\title{
O uso do sistema de segurança cruzado como ferramenta para notificação de incidente: um relato de experiência
}

\author{
Using the cross-security system as a tool for incident notification: an experience report \\ Uso del sistema de seguridad cruzada como herramienta para la notificación de \\ incidentes: un informe de experiencia
}

Leisson Domingues Pinheiro ${ }^{1^{*}}$, Paola Alejandra Valenzuela Reyes ${ }^{1}$, Gal Caroline Alho Lobão ${ }^{1}$.

\begin{abstract}
RESUMO
Objetivo: O estudo propõe implementar provas com o Safety Cross System como ferramenta inovadora para notificação de incidente. Relato de Experiência: Este estudo constitui um relato de experiência de caráter descritivo de um Centro integrado de inclusão e reabilitação do estado do Pará em relação a implementação de evidências em um sistema de notificação de incidentes que usa um calendário cruzado para registrar as notificações de incidentes permite uma metodologia visual para gerenciar a segurança do paciente de forma transparente, ou seja, o processo registra notificações de incidentes com cores diferentes para cada classificação. Considerações Finais: Algumas instituições adotam diferentes sistemas para melhorar os índices de notificações de incidente. O calendário cruzado é um sistema atraente, a equipe sente-se mais confortável em fazer parte do processo de notificação e análise, propondo melhorias de forma democrática aos processos de segurança do paciente.
\end{abstract}

Palavras chave: Segurança do paciente, Erros médicos, Saúde pública.

\begin{abstract}
Objective: The study proposes to implement evidence with the Safety Cross System as an innovative incident notification tool. Experience Report: This study constituted an account of the experience of a descriptive character of an Integrated Center of Inclusion and Rehabilitation of the State of Pará in relation to implementation of evidências in an incident notification system that uses a cross calendar to record incident reports. It allows a visual methodology. To manage patient insurance transparently, ou seja, or process records notifications of incidents with different cores for each classification. Considerations: Some institutions have different systems to improve the rates of incident reports. Or cross calendar and an attractive system, in addition to more comfort in the process of notification and analysis, I propose melodies democratically to the processes of patient safety.
\end{abstract}

Key worlds: Patient Safety, Mistakes Doctors, Public Health.

\section{RESUMEN}

Objetivo: El estudio propone implementar evidencia con el Sistema de Seguridad Cruzada como una herramienta innovadora de notificación de incidentes. Informe de experiencia: Este estudo constituye un relato de experiencia de carátula descrita del Centro integrado de inclusión y reactivación del estado del Pará en relación con la implementación de evidências en un sistema de notificación de incidentes que usa um calendário cruzado para registrador como notificaciones de incidentes habilitados en una metodología visual para gerenciar a segurança do paciente de forma transparente, ou seja, o proceso de registro de notificaciones

${ }^{1}$ Centro Integrado de Inclusão e Reabilitação. (CIIR/INDSH), Belém-PA. *E-mail: leisson.ona@bol.com.br 
de incidentes con núcleos diferentes para cada clasificación. Considerações finais: Algumas instituições adotam diferentes sistemas para melhorar os índices de notificações de incidente. O calendário cruzado é um system atraente, equipe sente-se mais comfortável em fazer parte do processso of notificação and análise, propondo melhorias de forma democrática aos processos de segurança do paciente.

Palabras clave: Seguridad del paciente, errores médicos, salud pública.

\section{INTRODUÇÃO}

A Organização Mundial de Saúde (OMS) estima que um em cada seis pacientes internados em hospitais é vítima de algum tipo de incidentes. No Brasil a incidência é de 7,6\% de pacientes acometidos por eventos adversos, sendo $66,7 \%$ são evitáveis. O que se torna importante a realização das notificações para que planos de melhorias possam ser implantados (CAPUCHO HC, et al., 2018).

Uma assistência prestada de forma insegura pode resultar em um evento adverso que resulta em gastos adicionais, aumento de permanência e dados expressivos de morbidade e mortalidade. As principais causas de eventos adversos são, por exemplo, erros de dosagem de medicamento ou aplicação de medicamento, uso incorreto de equipamento, infecções hospitalar, entre inúmeros outros casos. Esse fato é uma preocupação na atualidade. Importante que se trata de eventos que poderiam ser evitados e esses eventos são reflexo da qualidade da assistência (GOUVÊA CSD e RAVASSOS C, 2010).

A questão de segurança do paciente tem se tornado assunto de grande relevância nas organizações de saúde. Estudo realizado com notificações registradas no Sistema de Notificações para Vigilância Sanitária, em um período de 2 anos, o Brasil encontrou a ocorrência de 63.933 eventos adversos relacionados com a assistência à saúde, sendo desses, 417 com evolução para óbito (MAGALHES FHL, et al., 2019).

Em uma revisão sistemática foi avaliado um estudos de avaliação da ocorrência de eventos adversos em hospitais de sete países, demonstraram que a incidência de eventos adversos variou de 2.9 a 16,66 por 100 pacientes. Desse total de eventos adversos, 38\% estavam relacionados a procedimentos cirúrgicos e $19 \%$ a medicamentos. Outro estudo relacionado em 3 hospitais brasileiros a incidência de pacientes com evento adversos foi de $7,6 \%$ sendo que $35,2 \%$ dos eventos adversos estavam relacionados a procedimentos cirúrgicos e 5,6\% a medicamentos (ROMANO NS e MARQUES LFG, 2014).

Um evento adverso grave representa uma série de eventos adversos menores e representa a ponta de um iceberg. Cargas depreciativas do erro e defesa psíquica fazem que esses erros permaneçam escondidos, ou seja, sejam subnotificados ou esquecidos pelos profissionais da saúde (ALVES EDV, 2013). A ocorrência de incidente é um sério problema relacionado a segurança do paciente e a qualidade que dá assistência prestada (SIMAN AG e BRITO MJM, 2016).

O Ministério da Saúde institui em 2013 o Programa Nacional de Segurança do Paciente para um apelo aos profissionais de saúde e a população em geral, por uma atenção segura, livre de incidentes que possam ocasionar danos à saúde do indivíduo. Nesse contexto o clima de segurança percebido pelos profissionais é um ponto de partida para as organizações de saúde e há possibilidade de mensuração (TOSO GL, et al., 2016).

Diante desse cenário, ainda há receio e o medo entre os profissionais de saúde quando se aborda situações de erro, além do desconhecimento frente às intercorrências que possam ocorrer com o paciente e temor quanto a seu futuro profissional. Tudo isso resulta em muitas subnotificações ou não notificação dos erros (BOHOMOL E e RAMOS LH, 2007) O modelo de punição ainda está presente na concepção de muitos gestores e instituições e que acaba levando a nova recorrência dos incidentes de segurança (GERHARDT LM e UERBANETTO JS, 2013).

A cruz de segurança é uma metodologia de um programa de melhoria de qualidade para capacitar enfermeiros, parteiras e equipe multidisciplinar para otimizar a maneira de gerenciar o trabalho. Atualmente esse sistema é utilizado nacionalmente na Irlanda em 27 hospitais e vêm observando mudanças e experiências positivas dos pacientes. Muitas melhorias de segurança do paciente foram relatadas através do 
uso desse sistema, o valor do uso que envolve toda a equipe e incentivo a participação de toda a equipe, além de apresentar uma ferramenta simples e visual para discussão de planos de melhorias (QPS, 2014).

Buscou nesse estudo, relatar uma forma de operacionalizar um sistema para diminuir esses sentimentos, implantando uma metodologia para o gerenciamento das notificações de incidentes, tornando o ambiente assistencial mais seguro. O estudo propõe relatar a experiência sobre implementar provas com o sistema cruzado de segurança (Safety Cross System) como ferramenta inovadora para notificação de incidente.

\section{RELATO DE EXPERIÊNCIA}

Este estudo constitui um relato de experiência de caráter descritivo de um Centro integrado de inclusão e reabilitação do estado do Pará (CIIR) em relação a implementação de evidências em um sistema de notificação de incidentes que usa um calendário cruzado para registrar os eventos. Permite uma metodologia visual para gerenciar a segurança do paciente de forma transparente, ou seja, o processo registra notificações de incidentes com cores diferentes para cada classificação. Os resultados são divulgados diariamente, entre dia 1 a 31 do mês vigente e discutidos entre o Núcleo de Qualidade e segurança do paciente (NQSP) e a equipe multiprofissional (médicos, enfermeiros, técnicos de enfermagem, fisioterapeutas, terapeutas ocupacionais, psicólogos, assistentes sociais e nutricionistas, entre outros) (Figura 1).

Figura 1 - Quadro demonstrativo do Safety Cross System.

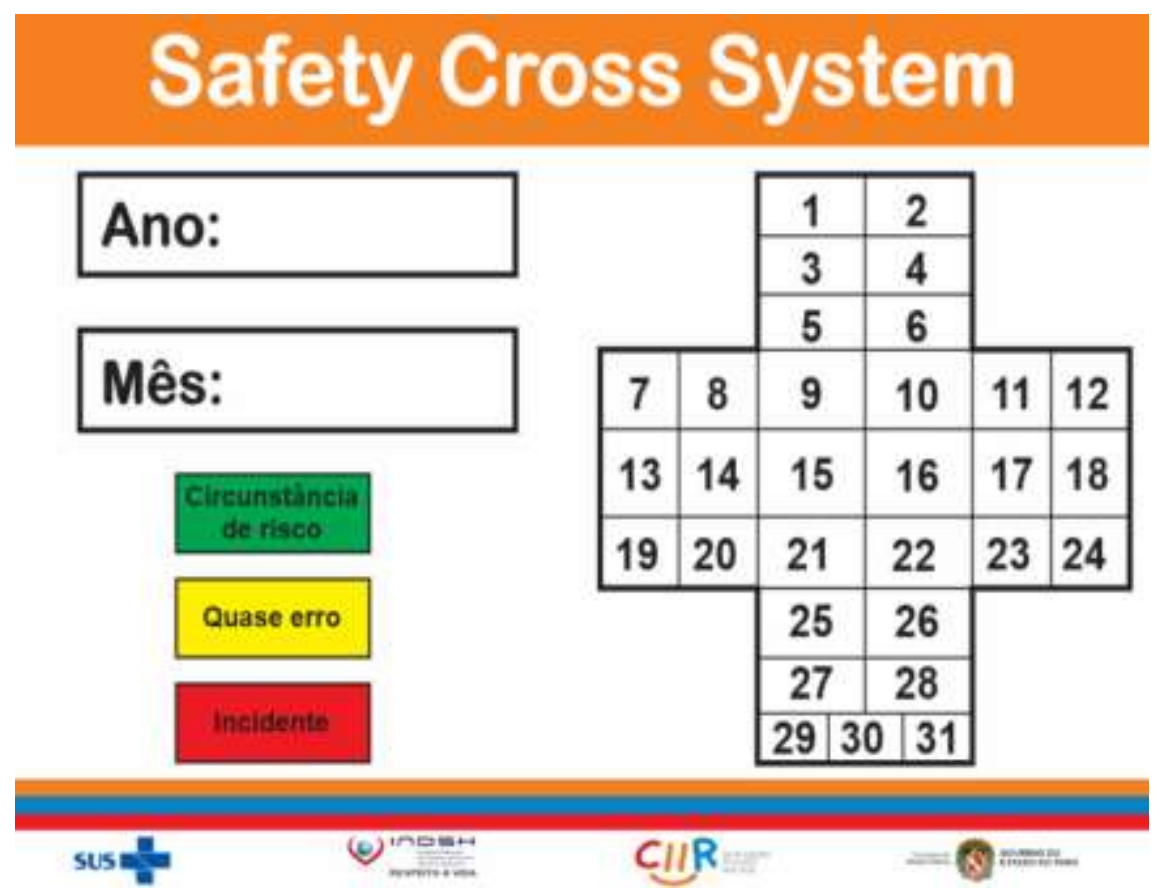

Fonte: Pinheiro LD, Reyes PAV, Lobão GCA, 2019.

As notificações são lançadas no quadro com as cores correspondente, sendo verde - circunstância de risco (situação que houve potencial significativo de dano desnecessário, mas não ocorreu um incidente), amarelo - quase erro (incidente que não atinge o paciente), vermelho - incidente (incidente que ocorreu a um paciente com ou sem danos).

Após lançamentos dos incidentes no quadro, a equipe multiprofissional juntamente com NQSP realizam as discussões das notificações de incidentes, relatando todos os fatos ocorridos e abrindo as discussões coletivas para implantar os planos de melhorias par cada incidente registrado. Importante perceber a participação democrática de todos os profissionais aplicando o conhecimento focado na segurança do 
paciente, habilidades, os valores diário, trabalho em equipe e o reconhecimento. Todos as reuniões têm periodicidade de 10 a 10 dias e são registrados a lista de presença dos participantes.

Nesse sentido, o Safety Cross System criou um metodologia mediadora para maior atenção da equipe e uma gestão transparente das notificações de incidentes dentro da organização. A metodologia apresentou uma ferramenta de motivação entre as equipes e incentivo às práticas assistenciais mais seguras.

No primeiro momento a equipe já demostrou interesse necessária ao conteúdo, despertando seriedade da atividade e seu propósito. Com o passar do tempo e alinhamento da transmissão das informações, o entendimento da equipe começou a ser observados em relação aos colaboradores propor ações mais efetivas para melhoria dos processos.

O emprego desse sistema tornou um instrumento importante para monitoramento e desmistificação das notificações de incidentes na instituição, aumentando o número de registro gradativamente entre o mês de março a agosto de 2019.

Os efeitos das mudanças foram observados, sendo que em março 15 registros, abril 9 (nove), maio 15 (quinze), junho 10 (dez), julho10 (dez), agosto 9 (nove). Sendo assim, visualiza-se que houve uma adesão da equipe quanto aos registros, que é uma das práticas de segurança do paciente. Importante que em tempo real esses resultado são apresentados e discutidos de forma democrática os planos de ações para melhoria da segurança do paciente. A equipe passou a notificar os incidentes com mais frequência e a partir disso contribuir com o monitoramento e aprimorar as práticas assistenciais com qualidade e segurança do paciente.

\section{DISCUSSÃO}

O estudo buscou relatar a forma de operação do sistema para o gerenciamento das notificações de incidentes e os resultados observados com o sistema cruzado de segurança, tornando o ambiente assistencial mais seguro.

Um estudo realizado em um hospital de ensino, foi observado que o sistema de notificações informatizados para notificação de incidentes em saúde apresentou uma maior qualidade de informações em comparação a notificação manuscrita, que apresentou ilegibilidade no momento de análise. O sistema informatizado favoreceu relatos espontâneos mais qualificados, ampliando a segurança do paciente no hospital (CASSIANI SHB, et al., 2010).

Em outro estudo realizado em um hospital universitário, avaliou a utilização deum boletim para realização de notificação de ocorrências assistenciais, o que viabilizou a utilização por auxiliares e técnicos de enfermagem. Esse recurso possibilita um acompanhamento dos eventos adversos associado a um recurso de comunicação, apresentando importante para a segurança do paciente (PAIVA MCMP, et al., 2010).

As notificações voluntárias sejam informatizadas ou manuscritas são essenciais para a construção da aprendizagem que faz parte da cultura de segurança do paciente. As notificações informatizadas sejam mais vantajosas por apresentar maior qualidade dos relatos, especialmente quanto a classificação e descrição da gravidade do incidente, descrição do paciente, elegibilidade, rasuras e ampliação de participação de profissionais (CAPUCHO HC, 2012).

Foi avaliado a efetividade dos incidentes on-line em um centro hospitalar de Lisboa central, onde foram constituídos 450 grupos envolvendo mais de 1350 profissionais para análise de incidentes e permitiu uma maior análise do circuito dos relatos de incidentes. Cada grupo é notificado do relato através de email e pode fazer a sua análise, avaliando e recomendando melhoria, promovendo uma cultura e de prevenção (TRINDADE L e RAMOS S, 2013).

Foi observado o baixo número de notificações pode estar relacionado ao sistema adotado em uma instituição de grande porte, filantrópica que atende aos usuários do Sistema Único de Saúde, onde o profissional que notifica o incidente precisa ser identificado. Os notificadores despertam o sentimento de represálias (LORIZINI E, et al., 2014). 
A falta de tradição dos profissionais de saúde em notificar ou o reconhecimento de eventos refleti negativamente na competência. A subnotificação pode estar relacionada também em outros fatores, como, por exemplo, falhas durante o ensino médico, ações poucas expressiva dos conselhos de classe na vigilância da qualidade prescrição, inércia dos pacientes e seus familiares na luta por direitos frente à iatrogenia (OLIVEIRA JR, et al.,2013).

A inovação é bastante rica e abrangente do que conceito de mudanças, renovação ou a reforma. Requer uma ação persistente. A metodologia inovadora cria um caminho característico para maior atenção e encoraja a participação de todos no processo de ensino e aprendizagem (BUSS CS, et al., 2017).

Conforme relatório do time de cuidados, um programa de melhoria de qualidade que visa capacitar a equipe multidisciplinar de 27 hospitais na Irlanda, vem apresentando resultado como desenvolvimento da cultura de segurança, análise regulares da equipe em relação aos dados como ferramenta de melhoria sustentada, concentração direta na assistência, incentivo para participação de toda a equipe, aumento de chance de sucesso de ações preventivas de eventos adversos (QUALITY e SAFETY, 2014).

Um programa de intervenções educativas para estimular às notificações voluntárias podem trazer benefícios para instituição, aumentando o número. Em um serviço de gerenciamento de risco de um hospital de ensino da Rede sentinela no interior de São Paulo apresentou um aumento de $29 \%$ em um período de um ano, com intervenções educativas (PRIMO LP e CAPUCHO HC, 2011).

O evento adverso evitáveis representa um problema indicativo da qualidade da assistência, gera sofrimento dos pacientes e colaboradores. Representa uma preocupação da Organização mundial de saúde que busca desenvolver metodologias para a detecção (LANZILLOTTI LS, et al., 2013).

A segurança do paciente depende de empenho político e organizativo dos profissionais e dos pacientes, bem como todos os intervenientes do processo com o sistema de saúde. Mudanças são importantes para implantar e promover cultura de segurança desde a entrada até a saída do paciente. Apesar de profissionais realizar notificações, apenas $28 \%$ dos médicos participou das notificações em um estudo na rede de médicos sentinela em Portugal. Tal fato não é realidade somente do país a baixa adesão desses profissionais (RIBAS MJ, 2010).

As notificações durante a prestação de cuidados na saúde é considerado uma ferramenta estratégica eficaz para gerenciar, diminuir e controlar a ocorrência dos incidentes, além de planejamento de ações de educação continuada, direciona ações para melhoria nas organizações de saúde e potencializa as melhorias dos serviços e avaliação da qualidade da assistência (REGINA M, et al.,2014).

As funções atraentes desse sistema utilizado no CIIR, a equipe sente-se mais confortável em fazer parte do processo de notificação e análise, propondo melhorias de forma democrática aos processos de segurança do paciente. O sistema é de grande praticidade, fácil manuseio e baixo custo, além da aceitação da equipe assistencial. Portanto uma opção para trabalhar com as notificações de incidentes, seja incidentes analisados por setores diferentes, como, por exemplo clínica médica, clínica cirúrgica, unidades de terapia intensiva, ambulatórios, serviço auxiliar de diagnóstico e terapia, entre outros ou analisados de uma forma macro nas organizações de saúde.

\section{REFERÊNCIAS}

1. ALVES EAV. Segurança do paciente: erro à prevenção do risco.Cad IberAmer.Direito.Sanit.2013;2(2):723-733.

2. BOHOMOL E, RAMOS LH. Erro de medicação: importância da notificação no gerenciamento da segurança do paciente.2007;60(1):32-36.

3. BUSS CS, MACKEDANZ LF. O ensino através de projetos com metodologia ativa de ensino e de aprendizagem. Revista Thema:2017;14(3): 122-131.

4. CAPUCHO HC, et al. Segurança do paciente: Comparação entre notificações voluntárias manuscritas e informatizadas sobre incidentes em saúde.Rev Gaúcha Enferm.2013;31(1):164-172. 
5. CAPUCHO HC. Sistemas manuscrito e informatizado de notificações voluntária de incidente em saúde como base para cultura de segurança do paciente. Universidade São Paulo. Dissertação (Doutorado em Ciência) - Escola de Enfermagem de Ribeirão Preto. São Paulo, 2012;155 p.

6. CASSIANI SH, et al. Segurança do paciente: comparação entre notificações voluntárias manuscritas e informatizadas sobre incidentes em saúde.Rev Gaúcha Enferm.2013;34(1):164-172.

7. GERHARDT LM, UERBANETTO JS. Segurança do paciente na tríade assistência ensino e pesquisa.Rev Gaúcha Enferm.2013;31(3):8-9.

8. GOUVÊA CSD, TRAVASSO C. Indicadores de segurança do paciente para hospitais de pacientes agudos: Revisão sistemática.Cad Saúde Pública.2010;26(6): 1061-1078.

9. LANZILLOTTI LS, et al. Eventos adversos e outros incidentes na unidade de terapia intensiva neonatal. Ciência e Saúde coletiva.2013:937-946.

10. LORENZINI E, et al. Segurança do paciente: análise dos incidentes notificados em um hospital do sul do Brasil. Ver Gaúcha Enferm.2014;35(2):121-127.

11. MAGALHÃES FHL, et al. Clima de segurança do paciente em um hospital de ensino. Rev Gaúcha de Enferm.2019; 40:1-7.

12. PAIVA MCMP, et al. Eventos adversos: análise de um instrumento de notificação utilizado no gerenciamento de enfermagem. Revista da Escola de Enfermagem.2010;44(2):287-294.

13. PRIMO LP, CAPUCHO HC.Intervenções educativas para estímulo a notificação voluntárias em um hospital de ensino na rede sentinela. Revista Brasileira de farmácia hospitalar e serviços de saúde.2011;2(2):26-30.

14. QPS. Abouth the HSE Quality and Safety Directores: the quality and Patient Safety.Win.2014; $21: 10$.

15. QUALITY e SAFETY. The safety cross system: simple and effective. Disponível em: https://www.inmo.ie/tempDocs/QS_PAGE30dec13-jan14.pdf.Acesso em: 20 out.2019.

16. ROMANO NS, MARQUES LFGM. Estratégias para a segurança do paciente no processo de uso de medicamento após alta hospitalar. Revista de saúde Coletiva.2014;24(2):401-420.

17. REGINA M, et al. Incidentes: instrumento de gerenciamento da assistência para a segurança do paciente em pronto socorro. Revista eletrônica trimestral de enfermagem.2014; 34:219-231.

18. RIBAS MJ. Eventos adversos em cuidados de saúde primários: promover uma cultura de segurança do paciente. Revista Port clin geral.2010; 26:585-589.

19. SIMAN AG, BRITO MJM. Mudanças na prática de enfermagem para melhorar a segurança do paciente. Rev Gaúcha Enferm.2016; 37:1-9.

20. TOSO GL, et al. Cultura de segurança do paciente em instituições hospitalares na perspectiva da enfermagem.2016;37(2):1-8.

21. TRINDADE L, RAMOS S. Incidente de segurança do doente. Porquê relatar? Tecno hospital.2013;10-16.

22. OLIVEIRA JR, et al. Eventos adversos notificados aos Sistema nacional de notificações para vigilância sanitária (NOTIVISA): Brasil, estudo descritivo no período 2006 à 2011. Epidemiologia Serviço Saúde.2013;22(4):671-678. 